\title{
Moisture Modes and the Eastward Propagation of the MJO
}

\author{
ADAM SOBEL \\ Department of Applied Physics and Applied Mathematics, and Department of Earth and Environmental Sciences, \\ and Lamont-Doherty Earth Observatory, Columbia University, New York, New York \\ ERIC MALONEY \\ Department of Atmospheric Science, Colorado State University, Fort Collins, Colorado
}

(Manuscript received 3 July 2012, in final form 6 September 2012)

\begin{abstract}
The authors discuss modifications to a simple linear model of intraseasonal moisture modes. Wind-evaporation feedbacks were shown in an earlier study to induce westward propagation in an eastward mean low-level flow in this model. Here additional processes, which provide effective sources of moist static energy to the disturbances and which also depend on the low-level wind, are considered. Several processes can act as positive sources in perturbation easterlies: zonal advection (if the mean zonal moisture gradient is eastward), modulation of synoptic eddy drying by the MJO-scale wind perturbations, and frictional convergence. If the sum of these is stronger than the wind-evaporation feedback-as observations suggest may be the case, though with considerable uncertainty - the model produces unstable modes that propagate weakly eastward relative to the mean flow. With a small amount of horizontal diffusion or other scale-selective damping, the growth rate is greatest at the largest horizontal scales and decreases monotonically with wavenumber.
\end{abstract}

\section{Introduction}

Most early theories that aimed to explain the existence and properties of the MJO treated it as some form of atmospheric equatorial Kelvin wave, modified by interaction of moist convection with the larger-scale flow. In recent years, it has become clear that convectively coupled Kelvin waves do exist, but that the MJO occupies a distinctly different region of the spectrum (Wheeler and Kiladis 1999). It has also been realized that moisture plays a greater role than it did in the early theories. MJO simulation in particular is improved when convection is made more sensitive to environmental moisture (e.g., Tokioka et al. 1988; Wang and Schlesinger 1999; Maloney and Hartmann 2001; Benedict and Randall 2009; Hannah and Maloney 2011; Kim et al. 2012). Studies with limited-domain cloud-resolving models have shown that, at least under mean conditions of large-scale ascent and relatively frequent deep

Corresponding author address: Adam Sobel, Columbia University, Dept. of Applied Physics and Applied Mathematics, $500 \mathrm{~W}$. 120th St., Rm. 217, New York, NY 10027.

E-mail: latex@ahs129@columbia.edu convection, moisture contains most of the memory in the atmosphere that can regulate convection on time scales longer than a few days (Tulich and Mapes 2010; Kuang 2010).

The idea has emerged that the MJO is a moisture mode. We gave our own definition of this term in a previous study (Sobel and Maloney 2012, hereafter SM12), in which we also proposed a particular very simple model of a moisture mode with the aim of capturing the essential features of the MJO. That model did not appear to be particularly successful. The only linear unstable modes were westward propagating. A nonlinear mode also discussed in SM12 differed substantially in structure from the MJO, having a shocklike jump in moisture at the leading edge of an active phase, whereas the actual MJO has a gradual buildup. Here, we consider extensions to this model to incorporate additional processes that have been hypothesized to be important to the $\mathrm{MJO}$, and which one might expect to cause eastward propagation. These processes include advection of a mean zonal moisture gradient by perturbation zonal winds, modulation of synoptic-scale transient eddy drying by the MJO-scale zonal wind, and frictional convergence. 


\section{Model framework}

\section{a. Basic equations}

As in SM12, the only atmospheric equation is one for column water vapor $W$, varying explicitly only in the zonal direction and time:

$$
\frac{d W}{d t}=-\tilde{M} P+E-(1-\tilde{M}) R+k_{w} \frac{\partial^{2} W}{\partial x^{2}} .
$$

Here $E, P$, and $R$ are surface evaporation, precipitation, and column-integrated radiative cooling, all expressed in units of millimeters per second while $W$ is in millimeters. Equation (1) is really a moist static energy (MSE) equation, but the tendency and horizontal advection of temperature are neglected (the weak temperature gradient approximation) so that the tendency of moist static energy depends only on the tendency of $W$. The quantity $\tilde{M}=M / M_{s}$ is the "normalized gross moist stability," and $k_{w}$ is a diffusivity in the zonal direction. Here we work only with the linearized model

$$
\frac{\partial W^{\prime}}{\partial t}+U \frac{\partial W^{\prime}}{\partial x}=-\tilde{M} P^{\prime}+E^{\prime}-(1-\tilde{M}) R^{\prime}+k_{w} \frac{\partial^{2} W^{\prime}}{\partial x^{2}},
$$

where primes represent deviations from a zonally uniform basic state and $U$ is a constant background zonal wind. As the simplest choice we set $\tilde{M}$ constant, but a possible parameterization of variations in it due to frictional convergence is considered below. Hereafter, we drop primes. As before, $P$ is a function of $W$, and $R$ a function of $P$. When linearized these can be written

$$
\begin{aligned}
& P=W / \tau_{c} \\
& R=-r P=-r W / \tau_{c},
\end{aligned}
$$

with $\tau_{c}$ a convective time scale on the order of a day and $r$ a dimensionless constant of order 0.1-0.2 (Bretherton and Sobel 2002; Su and Neelin 2002; Lin and Mapes 2004). Surface latent heat flux is parameterized as a function of wind speed:

$$
E=C_{u} u
$$

with $C_{u}$ positive; the basic-state surface wind is assumed westerly, so that positive zonal wind perturbations increase the wind speed while negative ones decrease it. We do not attempt to model the air-sea humidity difference. Column water vapor (our prognostic variable) is related only loosely to it, and observations and comprehensive simulations support the notion that wind variations are the dominant control on evaporation variations on the MJO time scale. Further details and justification of this choice are given in SM12.

The zonal wind perturbation $u$-needed to compute the surface latent heat flux perturbation $E$ (in the linear model, we do not consider advection by the perturbation wind)—is computed by a projection operator, similar to a Green's function:

$$
u(x, t)=\int G\left(x \mid x^{\prime}\right) P\left(x^{\prime}, t\right)(1+r) d x^{\prime} .
$$

As in, SM12 we use

$$
G\left(x \mid x^{\prime}\right)= \begin{cases}-A e^{-\left(x-x^{\prime}\right) / L}, & x>x^{\prime}, \\ 3 A e^{3\left(x-x^{\prime}\right) / L}, & x<x^{\prime}\end{cases}
$$

Here the length scale $L$ is the group velocity of free Kelvin waves divided by a damping time scale.

\section{b. New processes}

As noted by SM12, the single linear mode of the uncoupled version of the system is westward propagating (relative to the mean wind) for all wavenumbers $k$. We consider whether other atmospheric processes, not included by SM12, might lead to eastward propagation. One possibility that has been raised in recent work is that the dry air advection by synoptic eddies into the equatorial belt is modulated by the planetary-scale zonal wind field associated with the MJO in such a way as to cause eastward propagation. There is evidence from observations (Maloney and Dickinson 2003; Maloney 2009; Kiranmayi and Maloney 2011) as well as simulations (Andersen and Kuang 2012) that low-level MJO westerlies favor stronger synoptic-scale eddies, while MJO easterlies suppress them. The synoptic-scale eddies bring in dry subtropical air; labeling synoptic-scale perturbations by double primes, we can add this process to (1):

$$
\begin{aligned}
\frac{\partial W}{\partial t}+U \frac{\partial W}{\partial x}= & -\tilde{M} P+E-(1-\tilde{M}) R \\
& +k_{w} \frac{\partial^{2} W}{\partial x^{2}}-\frac{\partial}{\partial y} \overline{v^{\prime \prime} W^{\prime \prime}} .
\end{aligned}
$$

When these eddies are stronger there is an effective sink of moist static energy to the equatorial belt, while when the eddies are weaker there is an effective source, in a perturbation sense. The precise mechanism by which the MJO zonal winds modulate the smaller-scale synoptic eddies is not fully understood, but most likely involves barotropic conversion of some form along with associated diabatic feedbacks (Sobel and Maloney 2000; Hartmann and Maloney 2001; Maloney and Dickinson 
2003). A natural first attempt at including this process in a simple linear model is to represent it as a perturbation MSE source proportional to minus the MJO zonal wind:

$$
-\frac{\partial}{\partial y} \overline{v^{\prime \prime} W^{\prime \prime}}=-D u
$$

with $D$ a constant, taken positive. Thus, (8) becomes

$$
\frac{\partial W}{\partial t}+U \frac{\partial W}{\partial x}=-\tilde{M} P+\left(C_{u}-D\right) u-(1-\tilde{M}) R+k_{w} \frac{\partial^{2} W}{\partial x^{2}}
$$

Another potentially relevant linear process is zonal MSE advection in the presence of a background eastward MSE gradient, which would contribute a term of the form $-u \partial \bar{W} / \partial x$ to the right-hand sides of (2) and (8), and thus a contribution $-\partial \bar{W} / \partial x$ to $D$ in (10). A gradient of the right sign to give moistening in easterly anomalies is present in the Indian Ocean basin, where the drier air over the western Indian Ocean transitions to a moister regime as one moves eastward toward the Maritime Continent. Hsu and Li (2012) argue that this mechanism is important to MJO development in the Indian Ocean.

Another potentially relevant mechanism is frictional convergence (Wang 1988; Wang and Rui 1990; Hendon and Salby 1994; Moskowitz and Bretherton 2000). As frictional convergence will occur in easterlies, where deep convection tends to be suppressed, we expect that this mechanically forced near-surface convergence will be compensated by relatively shallow divergent outflow. The resulting profile of upward vertical motion will be bottom heavy and thus lead to negative gross moist stability, as has been shown to be the case in the observed MJO (Haertel et al. 2008). In a linear perturbation sense we may represent this as

$$
\tilde{M}=\tilde{\bar{M}}+\tilde{M}_{p} u^{\prime},
$$

with $\overline{\tilde{M}}$ the mean value of the normalized gross moist stability $\tilde{M}$, and $\tilde{M}_{p}$ a coefficient, taken positive, giving the variations associated with frictional convergence. If we also write $P=\bar{P}+P^{\prime}$, then linearization of the whole convergence term gives

$$
\tilde{M} P \approx \bar{M} P^{\prime}+\tilde{M}_{p} \bar{P} u
$$

whose form differs from that of the convergence term in (8) by an additional term $\tilde{M}_{p} \bar{P} u$. The latter has the same form as the RHS of (9), so we can consider this process to contribute a quantity $\tilde{M}_{p} \bar{P}$ to $D$.
The combined effect of perturbations in latent heat flux and the other processes described above-modulation of synoptic-eddy drying, zonal advection, and frictional convergence-is expressed by the quantity $C_{u}-D$ in (10). If that quantity is positive (as in SM12 where $D=0$ ), low-level westerlies are associated with an MSE source and easterlies with an MSE sink. If it is negative, the converse is true.

Based on both recent studies with reanalysis data (e.g., Kiranmayi and Maloney 2011) and general circulation models (Maloney 2009), it appears that the sum of terms including horizontal advection and vertical advection contributes about $10 \mathrm{~W} \mathrm{~m}^{-2}$ of MSE increase per $1 \mathrm{~m} \mathrm{~s}^{-1}$ of zonal-wind anomaly in MJO easterly regions when averaged about the equator. This total exceeds the magnitude of the surface flux anomalies by about a third, so that $C_{u}-D<0$. The reanalysis vertical and horizontal advection contributions are comparable, although vertical advection is somewhat larger, to an extent that depends on the reanalysis product (Kiranmayi and Maloney 2011). In the western Pacific warm pool, the synoptic-eddy advection mechanism dominates the advection by the anomalous wind across the mean zonal humidity gradient, whereas in the Indian Ocean the converse is true. The reanalysis MSE budgets contain relatively large analysis increments that are of the same order as the horizontal and vertical advection terms (Kiranmayi and Maloney 2011), limiting the precision with which these values can be constrained. An implication of our analysis is that tighter observational constraints on these various MSE sources would be valuable.

Assuming sinusoidal perturbations $W^{\prime}=\mathcal{W} e^{i(k x-c t)}$, with $\mathcal{W}$ a complex amplitude and $c$ a (potentially) complex phase speed, and substituting we obtain

$$
\begin{aligned}
i k(U-c) W= & \tau_{c}^{-1}\left[r-\tilde{M}(1+r)-k_{w} k^{2}\right] W \\
& +\left(C_{u}-D\right) u-k_{w} k^{2} W .
\end{aligned}
$$

As in SM12, $u$ is found from (6),

$$
u=\Gamma(k)(1+r) P=\Gamma(k)(1+r) \tau_{c}^{-1} W,
$$

with

$$
\Gamma(k)=\frac{4 A\left[2 k^{2} / L+i\left(3 k / L^{2}+k^{3}\right)\right]}{\left(9 / L^{2}+k^{2}\right)\left(1 / L^{2}+k^{2}\right)} .
$$

It follows that the phase angle by which $u^{\prime}$ lags $P^{\prime}$ is

$$
\alpha=\tan ^{-1}\left(\frac{3}{2 k L}+\frac{k L}{2}\right) .
$$



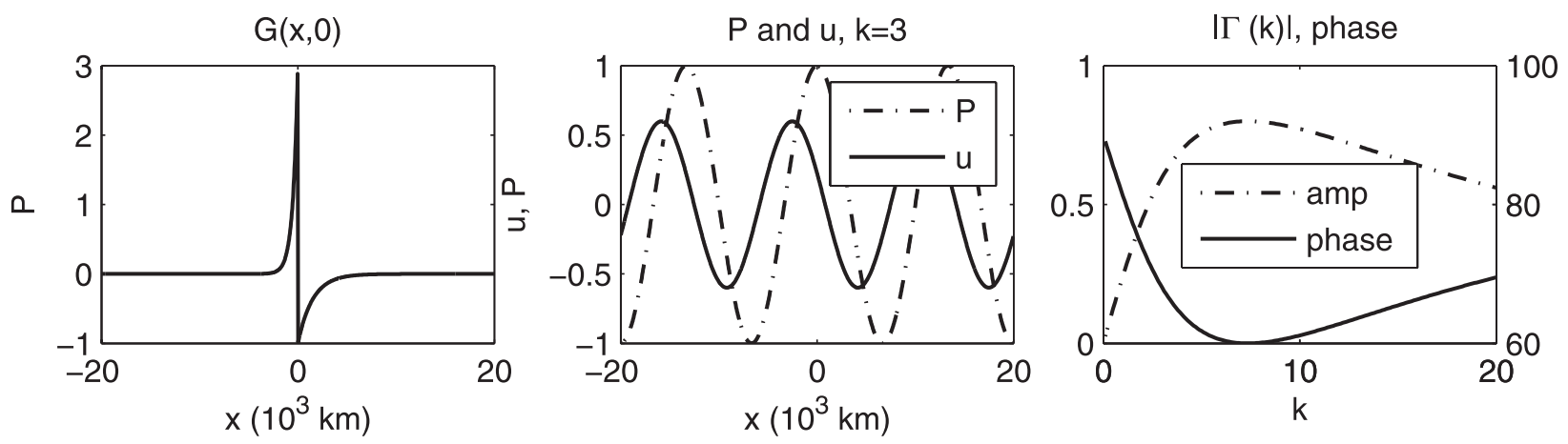

FIG. 1. (left) The projection function $G(x, 0)$-that is, the wind response to a $\delta$-function heating at $x^{\prime}=0$. (middle) The wind response to a sinusoidal heating of wavenumber 3 , together with that heating itself, as functions of $x$. (right) The amplitude and phase of the wind response function for sinusoidal heating $\Gamma(k)$, as a function of wavenumber $k$. The scale for amplitude is on the left axis, and the scale for phase $\left({ }^{\circ}\right)$ is on the right axis.

As the phase relationship between wind and water vapor is central to our discussion, in Fig. 1 we present several aspects of this relationship graphically; some aspects of this were mentioned in SM12. (See Table 1 of SM12 for all parameter values other than those stated here.) In the left panel the quantity $G(x \mid 0)$ is plotted (with $A=1$, for this panel only); this is the wind response to a $\delta$-function heating at the origin. In the middle panel we show the wind response to a sinusoidal heating of wavenumber $3\left[P=\cos \left(3 \pi x / L_{m}\right)\right.$ on our domain $-L_{m}<x<L_{m}$, with $L_{m}=2 \times 10^{4} \mathrm{~km}$; wavenumber 3 is chosen simply as an example. In the right panel we show the amplitude and phase of the function $\Gamma(k)$; positive phase means that wind lags precipitation, as it does here for all values of $k$. As $k \rightarrow \infty$ (and also as $k \rightarrow 0$ ), the amplitude $|\Gamma(k)| \rightarrow 0$ as the phase goes to $\pi / 2$. The wavenumber of maximum amplitude and minimum phase lag depends on $L$, and will be smaller than the value shown in Fig. 1 if $L$ is chosen greater than $1500 \mathrm{~km}$. At the minimum phase lag, the correlation of $W$ and MSE sources correlated with $u$ is greatest, yielding the strongest growth due to these terms if $C_{u}-D>0$ (SM12), or strongest damping by these terms otherwise.

The complex speed that results from solving (13) with (14) and (15) is

$$
c=U+\frac{\tilde{M}_{\mathrm{eff}}-\left(C_{u}-D\right) \Gamma+\tau_{c} k_{w} k^{2}}{i k \tau_{c}} .
$$

This is the same as (30) of SM12, except $C_{u}-D$ replaces $C_{u}$ and the horizontal diffusivity term is retained. The point of the present note is that the qualitative behavior of the solutions changes if $C_{u}-D<0$. The "effective gross moist stability," $\tilde{M}_{\text {eff }}=\tilde{M}(1+r)-r$, is a real constant, and so contributes only to growth (associated with the imaginary part of $c$ ) not propagation (the real part); further, as the growth rate is $\operatorname{Im}(c) k$, the contribution to the growth rate from the first term in the numerator of (17) is independent of $k$. Thus, all propagation comes from the second term, while all dependence on $k$ comes from the second and third terms. It was shown in SM12 that for $C_{u}>0$ and $D=0$, the second term induced growth for all $k$ but also westward propagation relative to the mean flow. The growth occurs because the latent heat flux perturbations have a nonnegative correlation with the MSE perturbations (since the phase lag between zonal wind and precipitation is always less than or equal to $\pi / 2$, and precipitation and moisture are in phase), the westward propagation because the latent heat flux always lags rather than leads moisture in longitude.

Now if the sign of this second term in (17) reverses-if the processes represented by $D$ depend on the zonal wind more strongly than the latent heat flux does, so that the net effect of easterlies is moistening and westerlies drying-both these effects reverse. The net effect of all these processes is then to cause weak eastward propagation (relative to the mean flow) but also to induce damping rather than growth. Observations, discussed above, suggest (with considerable uncertainty) that this may be the relevant regime for the Indian Ocean, and perhaps also the far western Pacific. In this regime the wind-evaporation feedback alone is still destabilizing, but not enough so to overcome the other wind-related feedbacks whose signs are opposite to it. Growth of disturbances can still occur, but only if $M_{\text {eff }}<0$.

The left panel of Fig. 2 shows the growth rate and phase speed as functions of $k$, for $r=0.15$, so that $\tilde{M}_{\text {eff }}=-0.035$, and $C_{u}-D=-2.5 \mathrm{~W} \mathrm{~m}^{-3} \mathrm{~s}$, and $k_{w}=0$. Because the only process that causes dependence on $k$ has reversed sign relative to SM12, the curves have shapes that are those shown in SM12 but upside down. Where the growth rate in SM12 reached a maximum at the intermediate wavenumber where the phase angle $\alpha$ minimized, and reached minima at $k=0$ and $k=\infty$, here the 

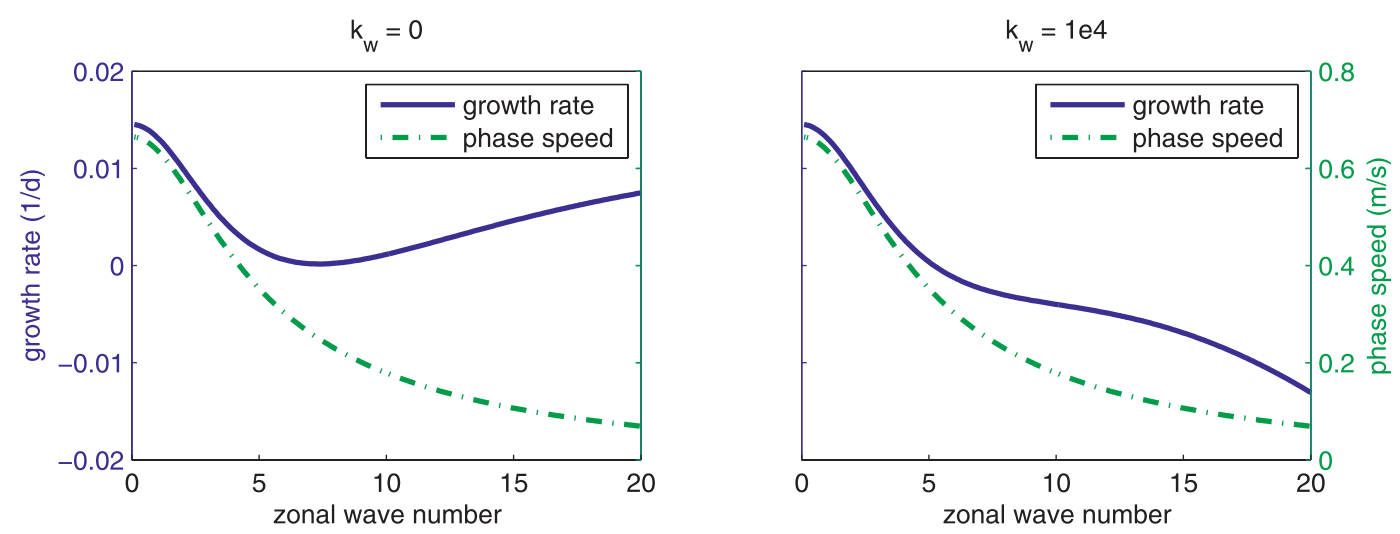

FIG. 2. Phase speed and growth rate for zonal moisture diffusivity (left) $k_{w}=0$ and (right) $k_{w}=10^{4} \mathrm{~m}^{2} \mathrm{~s}^{-1}$.

maxima are at $k=0$ and $k=\infty$, because the combined effect of the wind-evaporation feedback and the other wind-dependent processes vanishes there as the winds become in quadrature with $W$. The minimum growth rate is where $\alpha$ is a minimum because the combined effect of wind-dependent processes is damping at all finite $k$, but most strongly at that value. The phase speeds relative to the mean flow are weakly eastward for all $k$, most strongly at $k=0$. These phase speeds are smaller than the lowertropospheric mean westerlies in the Indian Ocean, Maritime Continent, and westernmost Pacific, so we expect the MJO propagation to be controlled by the mean westerly flow in the lower free troposphere.

The maximum growth rate as $k \rightarrow \infty$ for $k_{w}=0$ is not an "ultraviolet catastrophe" in that the growth rate does not blow up, but asymptotes to a constant value $\tilde{M}_{\text {eff }} \tau_{c}^{-1}$. Any positive $k_{w}$ (or any other scale-selective damping) will cause the asymptotic value to be zero instead; Fig. $2 \mathrm{~b}$ shows the curves for $k_{w}=10^{4} \mathrm{~m}^{2} \mathrm{~s}^{-1}$. A value of this order or greater might be loosely justifiable by the stochasticity of the convection. Also, if the term $-D u$ represents modulation of the collective effect of synoptic-scale eddies by the larger-scale wind associated with the MJO envelope, that implies an assumption that the MJO scale is itself larger than the synoptic scale. Thus, it makes no sense to allow the model to generate linearly unstable eddies of synoptic scale or smaller, and some form of scale-selective damping is justifiable to prevent growth at large $k$.

As stated above, instability occurs only if $\tilde{M}_{\text {eff }}<0$, meaning the sum of radiative-convective and convergence feedbacks is positive. In some models $\tilde{M}$ itself can be negative (e.g., Raymond and Fuchs 2009). Here, we have intentionally chosen $\tilde{M}_{\text {eff }}<0$ for the plots in Fig. 2 . The effect of varying this parameter is immediately apparent from (17); the growth rate contains a constant term that increases linearly with $-\tilde{M}_{\text {eff }}$, plus a $k$-dependent term that does not depend on $-\tilde{M}_{\text {eff }}$. The phase speed is entirely independent of $-\tilde{M}_{\text {eff }}$. The other important free parameter is $\tau_{c}$; both growth rate and phase speed scale as $\tau_{c}^{-1}$ for all $k$.

\section{Conclusions}

We have presented a brief analysis of a simple linear moisture-mode model. This is a modification of that in Sobel and Maloney (2012), including a simple representation of processes that have been proposed as contributing to eastward propagation of the MJO: modulation of synoptic-eddy drying by the MJO-scale zonal winds, advection of a mean zonal moisture gradient, and frictional convergence. All of these are crudely parameterized as sources of moist static energy that are proportional to minus the low-level zonal wind perturbation. Our primary conclusions are as follows:

(i) In order for eastward propagation of moisture modes to occur in this model, the other processes causing moistening in low-level easterlies must be stronger than the drying associated with suppression of surface fluxes in perturbation easterlies.

(ii) If the requirement for eastward propagation is met, this also implies that the net effect of all processes directly related to the low-level zonal wind on growth is negative: that is, damping. It is still the case that the surface wind-evaporation feedback by itself is destabilizing, but that destabilization is exceeded by the damping associated with the other zonal wind-related processes.

(iii) Eastward-propagating modes can only be unstable because of cloud-radiative feedback or gross moist instability. The effective gross moist stability, which captures the net effect of both, must be negative.

(iv) Absent any scale-selective damping, the growth rates of eastward-propagating unstable modes are 
maximized at the largest and smallest spatial scales in the system. There is a minimum in between at synoptic scales (precisely those where the maximum occurred in SM12).

(v) A modest horizontal diffusion, or other scaleselective damping, causes the growth rate to decrease monotonically with wavenumber so that the largest zonal scales are selected.

Acknowledgments. This research was sponsored by National Science Foundation Grant AGS-1062206 (AHS) AGS-1062161 (EDM) and AGS-1025584 (EDM), National Aeronautics and Space Administration Grant NNX09AK34G (AHS), National Oceanic and Atmospheric Administration Grants NA08OAR4320912 A6R (AHS) and NA08OAR4320893 \#7 and \#14 (EDM), and Office of Naval Research Grant N00014-12-1-0911 (AHS).

\section{REFERENCES}

Andersen, J. A., and Z. Kuang, 2012: Moist static energy budget of MJO-like disturbances in the atmosphere of a zonally symmetric aquaplanet. J. Climate, 25, 2782-2804.

Benedict, J. J., and D. A. Randall, 2009: Structure of the MaddenJulian oscillation in the superparameterized CAM. J. Atmos. Sci., 66, 3277-3296.

Bretherton, C. S., and A. H. Sobel, 2002: A simple model of a convectively coupled Walker Circulation using the weak temperature gradient approximation. J. Climate, 15, 2907-2920.

Haertel, P. T., G. N. Kiladis, A. Denno, and T. M. Rickenback, 2008: Vertical-mode decompositions of 2-day waves and the Madden-Julian oscillation. J. Atmos. Sci., 65, 813-833.

Hannah, W. M., and E. D. Maloney, 2011: The role of moistureconvection feedbacks in simulating the Madden-Julian oscillation. J. Climate, 24, 2754-2770.

Hartmann, D. L., and E. D. Maloney, 2001: The Madden-Julian oscillation, barotropic dynamics, and North Pacific tropical cyclone formation. Part I: Observations. J. Atmos. Sci., 58, $2545-2558$

Hendon, H. H., and M. L. Salby, 1994: The life cycle of the MaddenJulian oscillation. J. Atmos. Sci., 51, 2225-2237.

Hsu, P.-C., and T. Li, 2012: Role of the boundary layer moisture asymmetry in causing the eastward propagation of the MaddenJulian oscillation. J. Climate, 25, 4914-4931.

Kim, D., A. H. Sobel, A. D. D. Genio, Y. Chen, S. J. Camargo, M.-S. Yao, M. Kelley, and L. Nazarenko, 2012: The tropical subseasonal variability simulated in the NASA GISS general circulation model. J. Climate, 25, 4641-4659.
Kiranmayi, L., and E. D. Maloney, 2011: Intraseasonal moist static energy budget in reanalysis data. J. Geophys. Res., 116, D21117, doi:10.1029/2011JD016 031.

Kuang, Z., 2010: Linear response functions of a cumulus ensemble to temperature and moisture perturbations and implications for the dynamics of convectively coupled waves. J. Atmos. Sci., 67, 941-962.

Lin, J., and B. E. Mapes, 2004: Radiation budget of the tropical intraseasonal oscillation. J. Atmos. Sci., 61, 2050-2062.

Maloney, E. D., 2009: The moist static energy budget of a composite tropical intraseasonal oscillation in a climate model J. Climate, 22, 711-729.

— and D. L. Hartmann, 2001: The Madden-Julian oscillation, barotropic dynamics, and North Pacific tropical cyclone formation. Part I: Observations. J. Atmos. Sci., 58, 2545-2558.

— , and M. J. Dickinson, 2003: The intraseasonal oscillation and the energetics of summertime tropical western North Pacific synoptic-scale disturbances. J. Atmos. Sci., 60, 2153-2168.

Moskowitz, B. M., and C. S. Bretherton, 2000: An analysis of frictional feedback on a moist equatorial Kelvin mode. J. Atmos. Sci., 57, 1797-1816.

Raymond, D. J., and Z. Fuchs, 2009: Moisture modes and the Madden-Julian oscillation. J. Climate, 22, 3031-3046.

Sobel, A. H., and E. D. Maloney, 2000: Effect of ENSO and the MJO on western North Pacific tropical cyclones. Geophys. Res. Lett., 27, 1739-1742.

_ and - 2012: An idealized semi-empirical framework for modeling the Madden-Julian oscillation. J. Atmos. Sci., 69, 1691-1705.

Su, H., and J. D. Neelin, 2002: Teleconnection mechanisms for tropical Pacific descent anomalies during El Niño. J. Atmos. Sci., 59, 2694-2712.

Tokioka, T., K. Yamazaki, A. Kitoh, and T. Ose, 1988: The equatorial 30-60 day oscillation and the Arakawa-Schubert penetrative cumulus parameterization. J. Meteor. Soc. Japan, 66, 883-901.

Tulich, S., and B. E. Mapes, 2010: Transient environmental sensitivities of explicitly simulated tropical convection. J. Atmos. Sci., 67, 923-940.

Wang, B., 1988: Dynamics of tropical low-frequency waves: An analysis of the moist Kelvin wave. J. Atmos. Sci., 45, 20512065.

_ and H. Rui, 1990: Dynamics of the coupled moist KelvinRossby wave on an equatorial $\beta$-plane. J. Atmos. Sci., 47, 397-61.

Wang, W., and M. E. Schlesinger, 1999: The dependence of convective parameterization of the tropical intraseasonal oscillation simulated by the UIUC 11-layer atmospheric GCM. J. Climate, 12, 1423-1457.

Wheeler, M., and G. N. Kiladis, 1999: Convectively coupled equatorial waves: Analysis of clouds and temperature in the wavenumber-frequency domain. J. Atmos. Sci., 56, 374-399. 